\title{
Output Homogenization and Synchronization of Heterogeneous Nonlinear Multi-Agent Networks
}

\author{
Yanling Wei and Wim Michiels
}

\begin{abstract}
In this paper, we are concerned with the output synchronization of a group of heterogeneous agents with nonlinear dynamics. It is shown that via dynamic coupling, the output synchronization problem is transformed into a family of output homogenization and internal-model-based synchronization problems. In contrast to conventional approaches, the adopted approach does not rely on the generation of a specific reference trajectory, to be tracked by all agents. Based on this premise, we propose a distributed state-feedback protocol driving all the heterogeneous multi-agent individual systems onto a copy of a virtual reference signal. Invoking the results from the theory of output regulation and cooperative control, the state-feedback controller synthesis is conducted to achieve the output synchronization of heterogeneous multi-agent networks. Immediate extensions of the obtained results to observer-based control are also discussed. The effectiveness of the proposed output synchronization algorithm is verified through theoretical analysis and case studies.
\end{abstract}

\section{Index Terms}

Heterogeneous systems, Multi-agent networks, Output synchronization, Output regulation, State feedback, Observer-based feedback.

This work was supported by the project C14/17/072 of the KU Leuven Research Council, by the project G0A5317N of the Research Foundation-Flanders (FWO-Vlaanderen), and by the project UCoCoS, funded by the European Unions Horizon 2020 research and innovation programme under the Marie Sklodowska-Curie Grant Agreement No 675080.

Y. Wei and W. Michiels are with the Department of Computer Science, KU Leuven, Leuven, Belgium. Email: yanling.weidkuleuven.be (Y. Wei), wim.michiels@cs.kuleuven.be (W. Michiels) 


\section{INTRODUCTION}

The past two decades have witnessed a substantial growth of research interest in understanding and reshaping collaborative behaviors of distributed systems consisting of multiple agents or subsystems. This interest is primarily appealing from their various applications including the natural occurence of flocking and formation dynamics, formulation control of autonomous vehicles, distributed sensor networks, and power networks [1]-[11]. One common characteristic of this family of problems is that an asymptotic agreement among agents is achieved while the agents interact based on limited information about themselves and their surroundings. Much of the attention has been directed toward state/output synchronization in homogeneous networks, where the agent models are identical and they exchange information with their neighbors in terms of measurements of their own state/output relative to that of neighboring agents in the network [12], [13]. More generally, multi-agent networks in the presence of model uncertainties or external disturbances are intrinsically heterogeneous, where the agent dynamics are nonidentical. In contrast to homogeneous multi-agent systems, where the synchronization pattern is naturally defined by the common part of the agents' dynamics, the so-called "kernel" dynamics do not explicitly emerge for heterogeneous systems [14]-[16].

Therefore, the collaborative control of heterogeneous multi-agent networks is more chanllenging as agreement happens only upon proper compensation for the dynamics. This complexity appeals for the introduction of robust output regulation theory and the "internal model" principle to tackle the collaborative control problem [17]-[20]. In particular, the output synchronization problem for heterogeneous linear multi-agent networks was addressed in [17] via the classical robust output regulation theory.It is important to notice that the results in [17] are exclusively effective for heterogeneous networks with the same nominal dynamics, i.e., the inhomogeneity is only due to the presence of uncertainty on the agents' models. Towards a more general heterogeneous network, an internal model method was introduced in [20] to conduct the output synchronization analysis and design, where the key idea is to separately design a global homogeneous synchronization network and local output regulators. The commonly used scenarios for the synchronization of heterogeneous multi-agent networks can be categorized into two classes. For the first class, a reference trajectory is pre-specified [21], [22]. When the network achieves agreement, all the agent outputs approach the reference output trajectory. In particular, the existence of at least one agent is assumed which has access to the reference signal and shares 
this information to the whole group. This salient feature has been directly applied or adapted to networked output regulation problem, leader-follower configuration, and pinning control. In the other class of scenarios, there is no reference trajectory specified a priori [20], [23]. Specifically, the reference signal is not physically available to all the agents, and strategies to overcome this limitation were proposed via an internal model method. It has been shown that the former class requires more communication, possibly combined with structural restrictions for synchronization due to the augmented graph artificially reconstructed by the reference system and original multiagent network.

Compared to the developments for linear multi-agent systems, developments for nonlinear systems are at a quite early stage. Some interesting results have been available in various settings. For instance, the works on nonlinear multi-agent systems with homogeneous or heterogeneous autonomous agents in leader-follower networks were reported in [24]-[33], where a qualitative analysis on the synchronization mechanism was performed. However, the design of controller parameters achieving synchronization was not discussed. From the authors' best knowledge, few quantitative works in the literature address the output synchronization problem of heterogeneous nonlinear multi-agent networks in a numerical tractability manner, which motivates us for this study.

Based on the above observation, we consider the output synchronization problem for a group of heterogeneous multi-agent networks with nonlinear dynamics in a leader-free scenario, and we propose a systematic controller design procedure, which is tractable from a computational point of view. The proposed controller, which incorporated local reference generators, asymptotically regulates the individual agent dynamics to compensate the heterogeneity till the individual systems' outputs match the corresponding local references, and meantime, it aligns the reference trajectories to asymptotically synchronize through network collaboration. Technically, the Lyapunov method is used to analyse the dynamics of the closed-loop system, and the iterative algorithm is proposed for the controller design. Compared to the existing results, the contributions of this paper are twofold. Firstly, the designed controller only relies on the availability of relative output information instead of relative system's state/ controller's state information, which allows the operation of the synthesized controller in a much easier and more feasible manner. It leads for instance to a remarkable reduction of the communication burden, which is very relevant for the cooperative control of multi-agent systems. As a second contribution, the problem of finding a distributed state-feedback/observer-controller protocol that guarantees the output synchronization 
of heterogeneous multi-agent networks is formulated in terms of a feasibility problem of a set of matrix inequality constraints, which bridges quantitative works on the output synchronization problem of heterogeneous nonlinear multi-agent networks, and allows to synthesize the controller in a systematic manner.

The structure of the paper is as follows. In Section II, we introduce some preliminaries and formulate the problem of output synchronization for heterogeneous networks with nonlinear dynamics. In Section III, we derive an error-dynamics model for the study of output homogenization and synchronization, and the main design approach and its theoretical proof are discussed. In Section IV the effectiveness of the approach is illustrated by numerical simulations. Finally, the paper is concluded in Section V.

Notations. The notations that are used throughout this paper are standard. $\Re^{n \times m}$ stands for the set of $n \times m$ matrices. $\mathbf{I}_{n}$ and $\mathbf{0}_{m \times n}$ are used to denote the $n \times n$ identity matrix and $m \times n$ zero matrix, respectively, and $\mathbf{1}_{n}$ refers to a vector with all entries being 1 . The subscripts $n$ and $m \times n$ are omitted when the size is not relevant or can be determined from the context. $\operatorname{diag}\{\cdots\}$ denotes a block diagonal matrix. The notation $*$ is used to indicate the terms that are induced by symmetry.

\section{MODEL DESCRIPTION}

We consider a multi-agent network consisting of heterogeneous individual systems with nonlinear dynamics,

$$
\left\{\begin{array}{l}
\dot{x}_{i}(t)=A_{i} x_{i}(t)+B_{i} u_{i}(t)+E_{i} \phi_{i}\left(z_{i}(t)\right), \\
y_{i}(t)=C_{i} x_{i}(t), \\
z_{i}(t)=H_{i} x_{i}(t), \quad i \in \mathbb{V}:=\{1,2, \cdots, N\}
\end{array}\right.
$$

where $x_{i}(t) \in \Re^{n_{x i}}$ are the system states, $u_{i}(t) \in \Re^{n_{u i}}$ the inputs, $y_{i}(t) \in \Re^{n_{y}}$ and $z_{i}(t) \in \Re^{n_{z}}$, respectively, the measurement output and system output of agent $i$. The function $\phi_{i}(\cdot): \Re^{n_{z}} \rightarrow$ $\Re^{n_{z}}$ represents an unknown nonlinearity, which is assumed to be sector bounded, i.e.,

$$
\left[\phi_{i}\left(z_{i}\right)-S_{1} z_{i}\right]^{\top}\left[\phi_{i}\left(z_{i}\right)-S_{2} z_{i}\right] \leq 0, \forall z_{i} \in \Re^{n_{z}}, i \in \mathbb{V}
$$

where $\left\{S_{1}, S_{2}\right\} \in \Re^{n_{z} \times n_{z}}$ are specified real symmetric matrices satisfying $0 \leq S_{1} \leq S_{2}$. The interconnection topology among the agents (1) is represented by a weighted directed graph $\mathbb{G}=(\mathbb{V}, \mathbb{E})$, where $\mathbb{V}$ is the set of nodes, $\mathbb{V}:=\{1,2, \cdots, N\}$, and the set of edges $\mathbb{E} \subseteq \mathbb{V} \times \mathbb{V}$. An edge of $\mathbb{E}$ from node $i$ to node $j$ is denoted by $(i, j)$, and thus $\mathbb{E} \subseteq\{(i, j): i, j \in \mathbb{V}, j \neq i\}$. 
The weighted adjacency matrix of graph $\mathbb{G}$ is $\mathbb{A}=\left[a_{i j}\right]_{N \times N} \in \Re^{N \times N}$ with non-negative elements where $a_{i j}=0$ for $(j, i) \notin \mathbb{E}$ and $a_{i j}>0$ for $(j, i) \in \mathbb{E}$.

Throughout the paper it is assumed that the directed network has a directed spanning tree, which is equivalent to the property that Laplacian matrix $L \in \Re^{N \times N}$ has a simple eigenvalue zero, and all the other eigenvalues have positive real parts [34]. It is convenient to remark that for Laplacian $L$, there exists a Jacobian decomposition of the form $L=T^{-1} \Lambda T$, where $T=\left[\begin{array}{ll}r & W^{\top}\end{array}\right]^{\top}, T^{-1}=\left[\begin{array}{cc}\mathbf{1}_{N} & U\end{array}\right], W \in \Re^{(N-1) \times N}, U \in \Re^{N \times(N-1)}$, and $r \in \Re^{N}$ and $\mathbf{1}_{N}$ are, respectively, the left and right eigenvectors corresponding to the eigenvalue zero with $r^{\top} \mathbf{1}_{N}=1$. In addition, $\Lambda \in \Re^{N \times N}$ is a block-diagonal Jordan matrix, $\Lambda=\operatorname{diag}\left\{0, \Lambda_{1}, \cdots, \Lambda_{m}\right\}$.

The heterogeneity precludes the achievement of full state synchronization through mutual coupling. However, as we shall see it may be still possible to achieve synchronization of the ouputs via the introduction of an internal model scheme. We consider an output communication configuration, that is, agent $i$ can only receive output information from its neighbors, while its internal states (including system state and controller state) are not available to its neighbors. Specifically, we establish a distributed state-feedback control law with the general form,

$$
\left\{\begin{array}{l}
\dot{\hat{x}}_{i}(t)=A_{0} \hat{x}_{i}(t)+F_{i} \vartheta_{i}(t) \\
u_{i}(t)=K_{1 i} x_{i}(t)+K_{2 i} \hat{x}_{i}(t)+K_{3 i} \vartheta_{i}(t), \quad i \in \mathbb{V},
\end{array}\right.
$$

where $\hat{x}_{i}(t)$ is the dynamic compensator state, $\vartheta_{i}(t)$ the weighted sum of the relative outputs between the agent and its neighbors, i.e.,

$$
\vartheta_{i}(t):=\sum_{j=1}^{N} a_{i j}\left(y_{j}(t)-y_{i}(t)\right)
$$

and $F_{i}, K_{1 i}, K_{2 i}$, and $K_{3 i}$ are the matrices describing the protocol to be designed.

The intuition behind (2) is as follows. In the absense of coupling, i.e., for $\vartheta_{i}(t)=0, i=$ $1, \cdots, n$, each controller stabilizes the corresponding subsystem and regulates its output to a reference signal generated by the first equation in (2). The role of coupling is then to make sure that these reference signals are synchronized. This will eventually result in all outputs synchronizing with the output corresponding to a solution of (virtual) system

$$
\left\{\begin{array}{l}
\dot{\hat{x}}_{0}(t)=A_{0} \hat{x}_{0}(t), \\
y_{0}(t)=C_{0} \hat{x}_{0}(t)
\end{array}\right.
$$

where $\hat{x}_{0}(t) \in \Re^{n_{0}}$ and $y_{0}(t) \in \Re^{n_{y}}$. Here we assume that the pair $\left(A_{0}, C_{0}\right)$ is of appropriate dimensions and observable, which can be selected a priori or computed from the regulator equation in the next section. The dynamics of (3) are constrained to $\operatorname{Re}\left\{\lambda_{k}\left(A_{0}\right)\right\}=0, k=1, \cdots, n_{0}$. 
The preclusion of the stable mode (i.e., eigenvalues having negative real part) is natural because they do not contribute the persistent signal $y_{0}(t)$, but the exclusion of exponentially unstable modes is for technical reason, where stronger connected assumptions on the communication graph should be imposed [12]. Nevertheless, the autonomous system (3) can generate a general class of patterns including constant and/or harmonic series.

In this paper, the output synchronization behavior is elaborated in the following sense.

Definition 2.1. The group of agents (1) is said to achieve output synchronization if for all initial conditions $x_{i}(0)$ and $\hat{x}_{i}(0)$ there exists an initial condition $\hat{x}_{0}(0)$ such that $y_{0}(t)$ governed by the dynamics (3) and the output trajectories of all agents satisfy

$$
\lim _{t \rightarrow \infty} y_{i}(t)-y_{0}(t)=0, i=1, \cdots, N
$$

To summarize, the objective of the paper is to set up the distributed state-feedback control law (2) for each agent, with the network measurement $\vartheta_{i}(t)$, such that the group of closed-loop agents composed of (1) and (2) achieves output synchronization in the sense of Definition 2.1. Note that this problem is different from the leader-following principle since an external reference generator of the form (3) is not present and the initial condition of $\hat{x}_{i}(0)$ determining the output trajectory after synchronization depends on the initial state of the network.

\section{Conditions For Output Synchronization of Heterogeneous Networks}

In this section, we firstly provide an output synchronization criterion for heterogeneous nonlinear networks, where the output synchronization problem is transformed into the stability analysis problem of an interconnected system involving the output homogenization and the relative stability of the reference generators. Then the design of the distributed state-feedback control law is addressed and extensions to observer-based controllers are made.

\section{A. Output synchronization criterion for heterogeneous networks}

Before presenting our main result, some notations are first introduced as below,

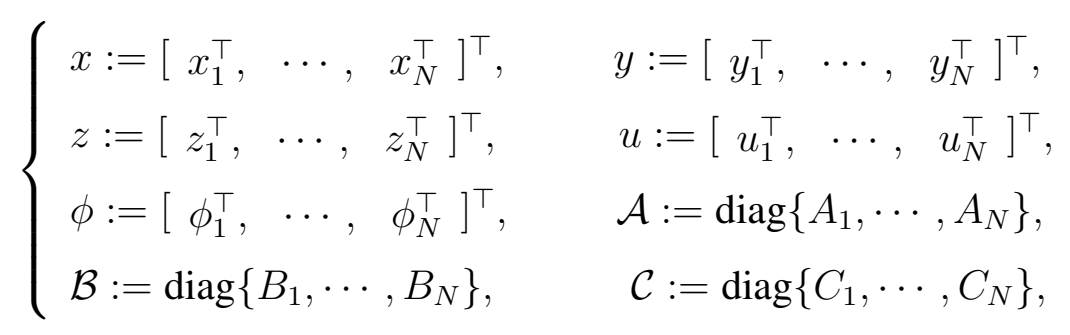




$$
\begin{cases}\mathcal{E}:=\operatorname{diag}\left\{E_{1}, \cdots, E_{N}\right\}, & \mathcal{H}:=\operatorname{diag}\left\{H_{1}, \cdots, H_{N}\right\}, \\ \mathcal{F}:=\operatorname{diag}\left\{F_{1}, \cdots, F_{N}\right\}, & \mathcal{K}_{1}:=\operatorname{diag}\left\{K_{11}, \cdots, K_{1 N}\right\}, \\ \mathcal{K}_{2}:=\operatorname{diag}\left\{K_{21}, \cdots, K_{2 N}\right\}, & \mathcal{K}_{3}:=\operatorname{diag}\left\{K_{31}, \cdots, K_{3 N}\right\} .\end{cases}
$$

By connecting the controllers in (2) to the agents in (1), the closed-loop system can be written in a compact form as,

$$
\left\{\begin{aligned}
\dot{\hat{x}}(t) & =\left(\mathbf{I}_{N} \otimes A_{0}\right) \hat{x}(t)+\overline{\mathcal{D}} x(t) \\
\dot{x}(t) & =\overline{\mathcal{A}} x(t)+\overline{\mathcal{B}} \hat{x}(t)+\mathcal{E} \phi(z) \\
y(t) & =\mathcal{C} x(t) \\
z(t) & =\mathcal{H} x(t)
\end{aligned}\right.
$$

where

$$
\left\{\begin{array}{l}
\overline{\mathcal{A}}:=\mathcal{A}+\mathcal{B} \mathcal{K}_{1}-\mathcal{B} \mathcal{K}_{3}(L \otimes \mathbf{I}) \mathcal{C} \\
\overline{\mathcal{B}}:=\mathcal{B} \mathcal{K}_{2} \\
\overline{\mathcal{D}}:=-\mathcal{F}(L \otimes \mathbf{I}) \mathcal{C}
\end{array}\right.
$$

Then conditions for output synchronization problem are provided by the following theorem.

Theorem 3.1. Let $\left(\Pi_{i}, \Gamma_{i}\right)$ be a solution pair to the regulator equation

$$
\left\{\begin{array}{l}
\Pi_{i} A_{0}=A_{i} \Pi_{i}+B_{i} \Gamma_{i} \\
C_{0}=C_{i} \Pi_{i} \\
\mathbf{0}=H_{i} \Pi_{i}, i=1,2, \cdots N
\end{array}\right.
$$

If there exists a positive definite matrix $P$, and a group of matrices $F_{i}, K_{1 i}$, and $K_{3 i}, i \in \mathbb{V}$, and a positive real scalar $\gamma$ such that

$$
\left[\begin{array}{cc}
\mathrm{He}\left\{\mathscr{A}^{\top} P\right\}-\gamma \chi^{\top} \mathcal{H}^{\top}\left(\mathbf{I} \otimes\left(S_{1} S_{2}+S_{2} S_{1}\right)\right) \mathcal{H} \chi & P \mathscr{E}+\gamma \chi^{\top} \mathcal{H}\left(\mathbf{I} \otimes\left(S_{1}+S_{2}\right)\right) \\
* & -2 \gamma \mathbf{I}
\end{array}\right]<0
$$

with $\chi:=\left[\begin{array}{ll}\mathbf{I} & \mathbf{0}\end{array}\right]$, and $K_{2 i}=\Gamma_{i}-K_{1 i} \Pi_{i}$, then the network of agents (1) with the aid of protocol (2) achieves output synchronization in the sense of Definition 2.1.

Proof. First, we also unify the notations

$$
\left\{\begin{array}{l}
\Pi:=\operatorname{diag}\left\{\Pi_{1}, \cdots, \Pi_{N}\right\} \\
\Gamma:=\operatorname{diag}\left\{\Gamma_{1}, \cdots, \Gamma_{N}\right\}
\end{array}\right.
$$

Then, according to Definition 2.1 the synchronization error of agents reads

$$
\begin{aligned}
e_{y}(t) & =y(t)-\mathbf{1}_{N} \otimes y_{0}(t)=\mathcal{C} x(t)-\mathbf{1}_{N} \otimes C_{0} \hat{x}_{0}(t) \\
& =\mathcal{C} x(t)-\left(\mathbf{I}_{N} \otimes C_{0}\right) \hat{x}(t)+\left(\mathbf{I}_{N} \otimes C_{0}\right) \hat{x}(t)-\mathbf{1}_{N} \otimes C_{0} \hat{x}_{0}(t) \\
& =\mathcal{C}(x(t)-\Pi \hat{x}(t))+\left(\mathbf{I}_{N} \otimes C_{0}\right)\left(\hat{x}(t)-\mathbf{1}_{N} \otimes \hat{x}_{0}(t)\right),
\end{aligned}
$$


where we utilize the condition $C_{0}=C_{i} \Pi_{i}$ in the regulator equation (5). Thus, if we can show that the first term vanishes along the solutions of the output homogenization, i.e., $\lim _{t \rightarrow \infty}(x(t)-$ $\Pi \hat{x}(t))=0$, and the second term vanishes along the synchronization of $\hat{x}_{i}(t), i \in \mathbb{V}$ and some choice of $\hat{x}_{0}(0)$ depending on the initial condition of the network, we can conclude the output synchronization of the underlying networks (1) in the framework of protocol (2).

For the former term $e_{x}(t):=x(t)-\Pi \hat{x}(t)$ direct computations yield,

$$
\begin{aligned}
\dot{e}_{x}(t) & =\overline{\mathcal{A}} x(t)+\overline{\mathcal{B}} \hat{x}(t)-\Pi\left[\left(\mathbf{I}_{N} \otimes A_{0}\right) \hat{x}(t)+\overline{\mathcal{D}} x(t)\right]+\mathcal{E} \phi(z) \\
& =\overline{\mathcal{A}} x(t)+\overline{\mathcal{B}} \hat{x}(t)-\Pi \overline{\mathcal{D}} x(t)-\left[\left(\mathcal{A}+\mathcal{B} \mathcal{K}_{1}\right) \Pi+\mathcal{B} \mathcal{K}_{2}\right] \hat{x}(t)+\mathcal{E} \phi(z) \\
& =\left(\mathcal{A}+\mathcal{B} \mathcal{K}_{1}\right) e_{x}(t)-\left[\mathcal{B K}_{3}(L \otimes \mathbf{I}) \mathcal{C}+\Pi \overline{\mathcal{D}}\right] x(t)+\mathcal{E} \phi(z) \\
& =\left(\mathcal{A}+\mathcal{B} \mathcal{K}_{1}\right) e_{x}(t)-\left(\mathcal{B} \mathcal{K}_{3}-\Pi \mathcal{F}\right)(L \otimes \mathbf{I}) \mathcal{C} x(t)+\mathcal{E} \phi(z) \\
& =\left(\mathcal{A}+\mathcal{B} \mathcal{K}_{1}\right) e_{x}(t)-\left(\mathcal{B} \mathcal{K}_{3}-\Pi \mathcal{F}\right)\left(L\left(\mathbf{I}-\frac{1}{N} \mathbf{1}_{N} \mathbf{1}_{N}^{\top}\right) \otimes \mathbf{I}\right) \mathcal{C} x(t)+\mathcal{E} \phi(z) \\
& =\left[\mathcal{A}+\mathcal{B} \mathcal{K}_{1}-\left(\mathcal{B} \mathcal{K}_{3}-\Pi \mathcal{F}\right)(L \otimes \mathbf{I}) \mathcal{C}\right] e_{x}(t)-\left(\mathcal{B K}_{3}-\Pi \mathcal{F}\right)\left(L U \otimes C_{0}\right) e_{\hat{x}}(t)+\mathcal{E} \phi(z),
\end{aligned}
$$

where we used the conditions in the regulator equation (5). In addition, when defining the coordinate $e_{\hat{x}}(t):=\left(U^{\top} \otimes \mathbf{I}\right) \hat{x}(t)$, we have,

$$
\begin{aligned}
\dot{e}_{\hat{x}}(t) & =\left(U^{\top} \otimes \mathbf{I}\right)\left[\left(\mathbf{I} \otimes A_{0}\right) \hat{x}(t)+\overline{\mathcal{D}} x(t)\right] \\
& =\left(\mathbf{I} \otimes A_{0}\right) e_{\hat{x}}(t)-\left(U^{\top} \otimes \mathbf{I}\right) \mathcal{F}(L \otimes \mathbf{I}) \mathcal{C} x(t) \\
& =\left[\mathbf{I} \otimes A_{0}-\left(U^{\top} \otimes \mathbf{I}\right) \mathcal{F}\left(L U \otimes C_{0}\right)\right] e_{\hat{x}}(t)-\left(U^{\top} \otimes \mathbf{I}\right) \mathcal{F}(L \otimes \mathbf{I}) \mathcal{C} e_{x}(t),
\end{aligned}
$$

where we used, once again, the conditions of the regulator equation (5). Finally, according to the definition of error $e_{x}(t)$, we can re-express the system output $z(t)$ in terms of $e_{x}(t)$,

$$
z(t)=\mathcal{H} x(t)=\mathcal{H}\left(e_{x}(t)+\Pi \hat{x}(t)\right)=\mathcal{H} e_{x}(t)
$$

where we apply the condition $\mathcal{H} \Pi=0$ in (5).

Combining the conditions (9), (10), and (11) we arrive at,

$$
\left\{\begin{array}{l}
\dot{e}_{x}(t)=\left[\mathcal{A}+\mathcal{B} \mathcal{K}_{1}-\left(\mathcal{B} \mathcal{K}_{3}-\Pi \mathcal{F}\right)(L \otimes \mathbf{I}) \mathcal{C}\right] e_{x}(t)-\left(\mathcal{B} \mathcal{K}_{3}-\Pi \mathcal{F}\right)\left(L U \otimes C_{0}\right) e_{\hat{x}}(t)+\mathcal{E} \phi\left(\mathcal{H} e_{x}\right), \\
\dot{e}_{\hat{x}}(t)=\left[\mathbf{I} \otimes A_{0}-\left(U^{\top} \otimes \mathbf{I}\right) \mathcal{F}\left(L U \otimes C_{0}\right)\right] e_{\hat{x}}(t)-\left(U^{\top} \otimes \mathbf{I}\right) \mathcal{F}(L \otimes \mathbf{I}) \mathcal{C} e_{x}(t)
\end{array}\right.
$$

To analyze the stability of the latter system, we choose the Lyapunov function $V\left(e_{x}, e_{\hat{x}}\right)=$ $\left[\begin{array}{ll}e_{x}^{\top} & e_{\hat{x}}^{\top}\end{array}\right]^{\top} P\left[\begin{array}{ll}e_{x} & e_{\hat{x}}\end{array}\right]$ with $P>0$. Obviously, $V\left(e_{x}, e_{\hat{x}}\right)$ is positive definite and radially unbounded. Then the time derivative of $V\left(e_{x}, e_{\hat{x}}\right)$ along the trajectories of the error system (12) is equal to,

$$
\dot{V}\left(e_{x}, e_{\hat{x}}\right)=2\left[\begin{array}{ll}
e_{x}^{\top} & e_{\hat{x}}^{\top}
\end{array}\right]^{\top} P\left[\mathscr{A}\left[\begin{array}{ll}
e_{x} & e_{\hat{x}}
\end{array}\right]+\mathscr{E} \phi\left(\mathcal{H} e_{x}\right)\right]
$$


where

$$
\left\{\begin{array}{l}
\mathscr{A}:=\left[\begin{array}{cc}
\mathcal{A}+\mathcal{B} \mathcal{K}_{1}-\left(\mathcal{B K}_{3}-\Pi \mathcal{F}\right)(L \otimes \mathbf{I}) \mathcal{C} & -\left(\mathcal{B K}_{3}-\Pi \mathcal{F}\right)\left(L U \otimes C_{0}\right) \\
-\left(U^{\top} \otimes \mathbf{I}\right) \mathcal{F}(L \otimes \mathbf{I}) \mathcal{C} & \mathbf{I} \otimes A_{0}-\left(U^{\top} \otimes \mathbf{I}\right) \mathcal{F}\left(L U \otimes C_{0}\right)
\end{array}\right], \\
\mathscr{E}:=\left[\begin{array}{cc}
\mathcal{E}^{\top} & \mathbf{0}
\end{array}\right]^{\top} .
\end{array}\right.
$$

In addition, according to the sector bounded properties of $\phi\left(\mathcal{H} e_{x}\right)$ we have

$$
\left[\begin{array}{c}
e_{x} \\
\phi\left(\mathcal{H} e_{x}\right)
\end{array}\right]^{\top}\left[\begin{array}{cc}
\mathcal{H}^{\top}\left(\mathbf{I} \otimes\left(S_{1} S_{2}+S_{2} S_{1}\right)\right) \mathcal{H} & -\mathcal{H}\left(\mathbf{I} \otimes\left(S_{1}+S_{2}\right)\right) \\
* & 2 \mathbf{I}
\end{array}\right]\left[\begin{array}{c}
e_{x} \\
\phi\left(\mathcal{H} e_{x}\right)
\end{array}\right] \leq 0 .
$$

Following from the S-procedure, condition (6) implies that $\dot{V}\left(e_{x}, e_{\hat{x}}\right)$ is negative definite for all $e_{x}(t)$ and $e_{\hat{x}}(t)$ satisfying (12) and all sector bounded function $\phi(z)$. Therefore, the solvability of conditions (5) and (6) guarantees that the synchronization error system (12) is asymptotically stable, and

$$
\lim _{t \rightarrow \infty} e_{x}(t)=0, \quad \lim _{t \rightarrow \infty} e_{\hat{x}}(t)=0 .
$$

It has been shown in [37] that $\hat{x}_{i}(t)-\hat{x}_{j}(t) \rightarrow \mathbf{0}$ as $t \rightarrow \infty, \forall i, j=1,2, \cdots N$ if and only if $e_{\hat{x}}(t)=\left(U^{\top} \otimes \mathbf{I}\right) \hat{x}(t) \rightarrow \mathbf{0}$ as $t \rightarrow \infty$. This fact in turn implies that the trajectories $\hat{x}_{i}(t)$ of the reference generators embedded in (2) are asymptotically convergent to a common trajectory. In particular, letting $e_{x}:=\left[\begin{array}{lll}e_{x 1}^{\top}, & \cdots, & e_{x N}^{\top}\end{array}\right]^{\top}$, we can rewrite the weighted measurement $\vartheta_{i}(t)$ in terms of $e_{x i}(t)$ and $e_{\hat{x} i}(t)$ as follows,

$$
\begin{aligned}
\vartheta_{i}(t) & =\sum_{j=1}^{N} a_{i j}\left(y_{j}(t)-y_{i}(t)\right) \\
& =\sum_{j=1}^{N} a_{i j}\left[C_{j} x_{j}(t)-C_{i} x_{i}(t)\right] \\
& =\sum_{j=1}^{N} a_{i j}\left[C_{j}\left(e_{x j}(t)+\Pi_{j} \hat{x}_{j}(t)\right)-C_{i}\left(e_{x i}(t)+\Pi_{i} \hat{x}_{i}(t)\right)\right] \\
& =\sum_{j=1}^{N} a_{i j}\left[C_{j} e_{x j}(t)-C_{i} e_{x i}(t)\right]+\sum_{j=1}^{N} a_{i j} C_{0}\left[\hat{x}_{j}(t)-\hat{x}_{i}(t)\right],
\end{aligned}
$$

where we bear $C_{i} \Pi_{i}=C_{0}$ in mind. According to the conclusion in (14), we have $\lim _{t \rightarrow \infty} \vartheta_{i}(t)=$ 0 and thus the dynamics of individual agents

$$
\dot{\hat{x}}_{i}(t)=A_{0} \hat{x}_{i}(t)+F_{i} \vartheta_{i}(t), i \in \mathbb{V}
$$

in (2) would reach a common trajectory satisfying the differential equation $\dot{\hat{x}}_{0}(t)=A_{0} \hat{x}_{0}(t)$. Specifically, the solutions of systems (16) are in the form of,

$$
\hat{x}_{i}(t)=\mathrm{e}^{A_{0} t}\left(\hat{x}_{i}(0)+\int_{0}^{t} \mathrm{e}^{-A_{0} s} F_{i} \vartheta_{i}(s) \mathrm{d} s\right), i \in \mathbb{V} .
$$


According to the specification of eigenvalues of $A_{0}$, i.e., $\operatorname{Re}\left\{\lambda_{k}\left(A_{0}\right)\right\}=0, k=1, \cdots, n_{0}$, as discussed in Section II, we always have $\left\|\mathrm{e}^{-A_{0} s}\right\| \leq c_{0} s^{l}$, where $c_{0}$ and $l$ are constants determined by the eigenvalues of $A_{0}$ and their corresponding multiplicity and (generalized) eigenvectors. Meantime, through the conditions (15), (5) and (6) we have shown that $\vartheta_{i}(t)$ is exponentially convergent to zero, and thus the integral $\int_{0}^{t} \mathrm{e}^{-A_{0} s} F_{i} \vartheta_{i}(s) \mathrm{d} s$ in (17) is bounded for all $t \in[0, \infty)$. In this regard, as $t \rightarrow \infty$, the solutions of systems (16) asymptotically converge to

$$
\mathrm{e}^{A_{0} t}\left(\hat{x}_{i}(0)+\int_{0}^{\infty} \mathrm{e}^{-A_{0} s} F_{i} \vartheta_{i}(s) \mathrm{d} s\right), i \in \mathbb{V} .
$$

The condition $\lim _{t \rightarrow \infty} e_{\hat{x}}(t)=0$ in (14) and the expression in (18) imply that $\mu:=\hat{x}_{i}(0)+$ $\int_{0}^{\infty} \mathrm{e}^{-A_{0} s} F_{i} \vartheta_{i}(s) \mathrm{d} s$ is independent of $i \in \mathbb{V}$. Furthermore, the expression in (18) is also the solution of the virtual system (3) under the initial condition $\hat{x}_{0}(0)=\mu$, and the individual dynamics of $\hat{x}_{i}(t), i=1,2, \cdots N$ converge to the trajectory $\hat{x}_{0}(t)=\mathrm{e}^{A_{0} t} \mu$. Finally, it follows from (8) that the measurement outputs $y_{i}(t), i=1,2, \cdots N$ also achieve synchronization to the corresponding ouput signal $y_{0}(t)$. This completes the proof. $\diamond$

Remark 3.1. In accordance with the synchronization error equation (12), the output synchronization of heterogeneous networks relies on the stability of two coupled dynamical subsystems: the first corresponds to the output homogenization error $e_{x}(t)$ system with individual system output asymptotically matching the local reference generator output, and the second describes the evolution of the synchronization error $e_{\hat{x}}(t)$ system regarding the reference trajectories.

In what follows, some comments on the role and necessity of regulator equation (5) are formulated.

Remark 3.2. Equation (5) is the so-called regulator equation, which is essentially a Sylvester equation and instrumental for establishing output regulation. In [35] it has been shown that this Sylvester equation has a unique solution if $A_{0}$ and $A_{i}$ have no common eigenvalues. The third equation in (5) assures that the outputs $z_{i}(t)$ vanish at the moment that synchronization is achieved (see [36] for more details). This is key why a solution of linear system $\dot{\hat{x}}_{0}(t)=A_{0} \hat{x}_{0}(t)$ can be asymptotically tracked, while at the same time only the sector condition (13) on the nonlinearities is taken into account.

Remark 3.3. In addition, under any suitable controller that solves the linear output regulation problem, the trajectories of the closed-loop system from any initial states $x(0)$ and $\hat{x}(0)$ satisfy $\lim _{t \rightarrow \infty}(x(t)-\Pi \hat{x}(t))=0$. Meantime, the control input satisfies $\lim _{t \rightarrow \infty}\left(u(t)-\left(\mathcal{K}_{1} \Pi+\mathcal{K}_{2}\right) \hat{x}(t)\right)=$ $\lim _{t \rightarrow \infty}(u(t)-\Gamma \hat{x}(t))=0$. This means that when output synchrony is reached, the closed-loop 
system has an asymptotically attractive invariant subspace $\mathscr{S}$ on which $y_{i}(t)=y_{0}(t), x_{i}(t)=$ $\Pi_{i} \hat{x}_{i}(t)$, and $u_{i}(t)=\Gamma_{i} \hat{x}_{i}(t)$, for $i=1, \cdots, N$.

\section{B. Protocol design for output synchronization}

In light of the stability analysis criterion in Theorem 3.1, we shall describe in detail the basis to our quantitative analysis framework to solve the output synchronization problem for heterogeneous multi-agent networks. We notice that there exist product terms between the Lyapunov matrix and controller matrices in the condition (6), which is essentially a bilinear matrix inequality (BMI) and brings some difficulties in the solutions of output synchronization problem. To this end, we shall resort to a decoupling technique to separate the Lyapunov matrix from the controller gains. The result is summarized in the following theorem.

Theorem 3.2. Consider the network of systems (1), and let $\left(\Pi_{i}, \Gamma_{i}\right)$ be a solution pair to the regulator equation (5). Given a scalar $\delta>0$, if there exist positive-definite symmetric matrices $P$ and $X$, matrices $F_{i}, K_{1 i}$, and $K_{3 i}, i \in \mathbb{V}$, and a positive scalar $\gamma$ such that

$$
\left[\begin{array}{ccc}
-\delta X & \mathbf{I}+\delta \mathscr{A} & \delta \mathscr{E} \\
* & -\delta^{-1} P-\gamma \chi^{\top} \mathcal{H}^{\top}\left(\mathbf{I} \otimes\left(S_{1} S_{2}+S_{2} S_{1}\right)\right) \mathcal{H} \chi & P \mathscr{E}+\gamma \chi^{\top} \mathcal{H}\left(\mathbf{I} \otimes\left(S_{1}+S_{2}\right)\right) \\
* & * & -2 \gamma \mathbf{I}
\end{array}\right]<0
$$

$$
P X=\mathbf{I},
$$

with $\chi:=\left[\begin{array}{ll}\mathbf{I} & 0\end{array}\right]$, and $K_{2 i}=\Gamma_{i}-K_{1 i} \Pi_{i}$, then the network of multi-agents (1) with the aid of control law (2), achieves output synchronization with pattern determined by (3).

Proof. We rewrite the condition in (6) as,

$$
\left[\begin{array}{cc}
-\gamma \chi^{\top} \mathcal{H}^{\top}\left(\mathbf{I} \otimes\left(S_{1} S_{2}+S_{2} S_{1}\right)\right) \mathcal{H} \chi & \gamma \chi^{\top} \mathcal{H}\left(\mathbf{I} \otimes\left(S_{1}+S_{2}\right)\right) \\
* & -2 \gamma \mathbf{I}
\end{array}\right]+\operatorname{He}\left\{\left[\begin{array}{l}
P \\
\mathbf{0}
\end{array}\right]\left[\begin{array}{ll}
\mathscr{A} & \mathscr{E}
\end{array}\right]\right\}<0 .
$$

It is straightforward that there exists a sufficiently small positive scalar $\delta$ such that the following inequality renders (21),

$$
\delta\left[\begin{array}{ll}
\mathscr{A} & \mathscr{E} \\
\mathbf{0} & \mathbf{0}
\end{array}\right]^{\top}\left[\begin{array}{ll}
P & \mathbf{0} \\
\mathbf{0} & \mathbf{I}
\end{array}\right]\left[\begin{array}{cc}
\mathscr{A} & \mathscr{E} \\
\mathbf{0} & \mathbf{0}
\end{array}\right]+\operatorname{LHS}(21)<0
$$

where LHS(21) denotes the left hand side of the condition (21). In addition, for any matrices $P, A$ and a positive scalar $\delta$, simple manipulations lead to the equality as below,

$$
P A+A^{\top} P+\delta A^{\top} P A=(\mathbf{I}+\delta A)^{\top}\left(\delta^{-1} P\right)(\mathbf{I}+\delta A)-\delta^{-1} P .
$$


Using the latter condition, along with applying the Schur complement formula twice, we can readily derive the conclusion in (19) and (20). This completes the proof. $\diamond$

The inequality in (19) is a linear matrix inequality (LMI), in contrast to BMI condition (6) from the previous theorem. However, the set of feasable solutions is not convex, due to the nonlinear equality constraint $P X=\mathbf{I}$. To solve this non-convex problem we shall resort to the cone complementarity linearization (CCL) technique [38], which could cast the nonconvex conditions into a sequential optimization problem subject to LMI constraints. The key procedure of CCL algorithm is that if the LMI $\left[\begin{array}{cc}P & \mathbf{I} \\ * & X\end{array}\right] \geq 0$ is feasible with the $n \times n$ matrix variables $P>0$ and $X>0$, then $\operatorname{Trace}(P X) \geq n$, and $\operatorname{Trace}(P X)=n$ if and only if $P X=\mathbf{I}$. This brings us to the following equivalent problem.

\section{Problem:}

Minimise Trace $(P X)$ subject to the condition in (19) and

$$
\left[\begin{array}{ll}
P & \mathbf{I} \\
* & X
\end{array}\right] \geq 0
$$

This problem can be solved using the following algorithm, whose convergence to a local minimum has been shown in [38], [39]:

\section{Algorithm:}

Step 1. Find a set $\left(P^{(0)}, X^{(0)}, F_{i}^{(0)}, K_{1 i}^{(0)}, K_{3 i}^{(0)}, i \in \mathscr{I}\right)$ that satisfies the condition in (19) and (23). Set $q=0$.

Step 2. Solve the following LMI problem over the variables $P, X, F_{i}, K_{1 i}, K_{3 i}$, and $\gamma$,

$$
\text { Minimise Trace }\left(P^{(q)} X+P X^{(q)}\right) \text { subject to (19) and (23). }
$$

Set $P^{(q+1)}=P$ and $X^{(q+1)}=X$.

Step 3. Substitute the gains $F_{i}, K_{1 i}$, and $K_{3 i}$ obtained in Step 2 into (6) and if the LMIs in (6) are feasible with respect to the variable $P$, then exit. Otherwise, set $q=q+1$, and go to Step 2.

Remark 3.4. The connection between the theorems is as follows. Theorem 3.1 establishes a stability analysis criterion for the output synchronization error system, expressed in terms of bilinear matrix inequalities. With some simple algebraic manipulations, these inequalities are transformed into linear inequalities with a simplifying nonlinear equality constraint, as formulated in Theorem 3.2. The latter is at the basis of a tractable sequential optimization problem with 
LMI constraints for the controller design. Compared to existing results, which are generally developed from the leader-following principle, the leader-free results in Theorem 3.1 do not require to exchange the controller's state. The latter property facilitates the execution of the developed controller in a much easier and more feasible way. Moreover, it can also reduce the communication burden, which would be important in the context of cooperative control of multi-agent systems. The communication reduction originates from two aspects: (i) the designed controller avoids the exchange of information on the controller's state, and (ii), instead of relative state information, the synthesized controller merely relies on the relative output information. Therefore, the elaborate control protocol is more attractive in practice.

\section{Extensions to observer-based control for output synchronization}

It is noted that the aforementioned control protocol is constructed based on the internal state of an agent $i$. However, in many practical situations, the internal states are not measurable, and for this case, an observer-based controller would be considered as an alternative. In particular, we consider the observer-controller,

$$
\left\{\begin{array}{l}
\dot{\xi}_{i}(t)=\left(A_{i}+G_{i} C_{i}\right) \xi_{i}(t)+B_{i} u_{i}(t)-G_{i} y_{i}(t) \\
u_{i}(t)=K_{1 i} \xi_{i}(t)+K_{2 i} \hat{x}_{i}(t)+K_{3 i} \vartheta_{i}(t), \quad i \in \mathbb{V}
\end{array}\right.
$$

together with the dynamic reference model $\dot{\hat{x}}_{i}(t)=A_{0} \hat{x}_{i}(t)+F_{i} \vartheta_{i}(t)$.

Similarly, we interconnect the controller in (24) to the agents in (1), which leads to the closed-loop system,

$$
\left\{\begin{array}{l}
\dot{\hat{x}}(t)=\left(\mathbf{I}_{N} \otimes A_{0}\right) \hat{x}(t)+\overline{\mathcal{D}} x(t), \\
\dot{x}(t)=\hat{\mathcal{A}}_{x} x(t)+\hat{\mathcal{A}}_{\xi} \xi(t)+\hat{\mathcal{A}}_{\hat{x}} \hat{x}(t)+\mathcal{E} \phi(z), \\
\dot{\xi}(t)=\hat{\mathcal{G}}_{x} x(t)+\hat{\mathcal{G}}_{\xi} \xi(t)+\hat{\mathcal{A}}_{\hat{x}} \hat{x}(t)
\end{array}\right.
$$

where

$$
\left\{\begin{array}{l}
\hat{\mathcal{A}}_{x}:=\mathcal{A}-\mathcal{B K}_{3}(L \otimes \mathbf{I}) \mathcal{C}, \\
\hat{\mathcal{A}}_{\xi}:=\mathcal{B} \mathcal{K}_{1}, \quad \hat{\mathcal{A}}_{\hat{x}}:=\mathcal{B} \mathcal{K}_{2}, \\
\hat{\mathcal{G}}_{x}:=-\mathcal{B} \mathcal{K}_{3}(L \otimes \mathbf{I}) \mathcal{C}-\mathcal{G C}, \\
\hat{\mathcal{G}}_{\xi}:=\mathcal{A}+\mathcal{G C}+\mathcal{B} \mathcal{K}_{1}, \\
\mathcal{G}:=\operatorname{diag}\left\{G_{1}, \cdots, G_{N}\right\} .
\end{array}\right.
$$

The following statement applies to the output synchronization of heterogeneous networks (1) driven by the observer-controller (24), and its proof is given in the Appendix. 
Corollary 3.1. Given a pair parameter $\left(\Pi_{i}, \Gamma_{i}\right)$ satisfying (5), if there exists a positive definite matrix $\bar{P}$, and a group of matrices $F_{i}, G_{i}, K_{1 i}$, and $K_{3 i}, i \in \mathbb{V}$, and a positive real number $\gamma$ such that

$$
\begin{aligned}
& {\left[\begin{array}{cc}
\operatorname{He}\left\{\bar{A}^{\top} \bar{P}\right\}-\gamma \bar{\chi}^{\top} \mathcal{H}^{\top}\left(\mathbf{I} \otimes\left(S_{1} S_{2}+S_{2} S_{1}\right)\right) \mathcal{H} \bar{\chi} & \bar{P} \overline{\mathscr{E}}+\gamma \bar{\chi}^{\top} \mathcal{H}\left(\mathbf{I} \otimes\left(S_{1}+S_{2}\right)\right) \\
* & -2 \gamma \mathbf{I}
\end{array}\right]<0,} \\
& K_{2 i}=\Gamma_{i}-K_{1 i} \Pi_{i}
\end{aligned}
$$

with $\bar{\chi}:=\left[\begin{array}{lll}\mathbf{I} & \mathbf{0} & \mathbf{0}\end{array}\right]$, and where

$$
\left\{\begin{array}{c}
\overline{\mathscr{A}}:=\left[\begin{array}{ccc}
\mathcal{A}+\mathcal{B} \mathcal{K}_{1}-\left(\mathcal{B} \mathcal{K}_{3}-\Pi \mathcal{F}\right)(L \otimes \mathbf{I}) \mathcal{C} & -\left(\mathcal{B K}_{3}-\Pi \mathcal{F}\right)\left(L U \otimes C_{0}\right) & -\mathcal{B K}_{1} \\
-\left(U^{\top} \otimes \mathbf{I}\right) \mathcal{F}(L \otimes \mathbf{I}) \mathcal{C} & \mathbf{I} \otimes A_{0}-\left(U^{\top} \otimes \mathbf{I}\right) \mathcal{F}\left(L U \otimes C_{0}\right) & \mathbf{0} \\
\mathbf{0} & \mathbf{0} & \mathcal{A}+\mathcal{G C}
\end{array}\right] \\
\overline{\mathscr{E}}:=\left[\begin{array}{ccc}
\mathcal{E}^{\top} & \mathbf{0} & \mathcal{E}^{\top}
\end{array}\right]^{\top},
\end{array}\right.
$$

then the network of multi-agents (1) with protocol (24) achieves output synchronization in the sense of Definition 2.1.

Now, by taking similar arguments as in the proof of Theorem 3.2, we have the following observer-based dynamic controller design results for heterogeneous networks (1).

Corollary 3.2. Consider the network of multi-agents (1) with the synchronization topology (24), and let $\left(\Pi_{i}, \Gamma_{i}\right)$ be a solution pair to the regulator equation (5). Given a scalar $\delta>0$, if there exist positive-definite symmetric matrices $\bar{P}$ and $\bar{X}$, matrices $F_{i}, G_{i}, K_{1 i}$, and $K_{3 i}, i \in \mathbb{V}$, and a positive scalar $\gamma$ such that

$$
\begin{aligned}
& {\left[\begin{array}{ccc}
-\delta \hat{x} & \mathbf{I}+\delta \overline{\mathscr{A}} & \delta \overline{\mathscr{E}} \\
* & -\delta^{-1} \bar{P}-\gamma \bar{\chi}^{\top} \mathcal{H}^{\top}\left(\mathbf{I} \otimes\left(S_{1} S_{2}+S_{2} S_{1}\right)\right) \mathcal{H} \bar{\chi} & \bar{P} \overline{\mathscr{E}}+\gamma \bar{\chi}^{\top} \mathcal{H}\left(\mathbf{I} \otimes\left(S_{1}+S_{2}\right)\right) \\
* & * & -2 \gamma \mathbf{I}
\end{array}\right]<0,} \\
& \bar{P} \bar{X}=\mathbf{I}, \\
& K_{2 i}=\Gamma_{i}-K_{1 i} \Pi_{i},
\end{aligned}
$$

then the network of agents (1) under the observer-based dynamic controller (24) achieves output synchronization with pattern determined by (3). 


\section{NUMERICAL EXPERIMENTS}

In this section, we present two numerical examples to demonstrate the effectiveness and advantages of the proposed method.

Example 4.1. We consider the output synchronization of systems (1) with system matrices specified as follows,

$\left[\begin{array}{c|c|c|c|c}A_{1} & B_{1} & C_{1} & E_{1} & H_{1} \\ \hline A_{2} & B_{2} & C_{2} & E_{2} & H_{2} \\ \hline A_{3} & B_{3} & C_{3} & E_{3} & H_{3} \\ \hline A_{4} & B_{4} & C_{4} & E_{4} & H_{4}\end{array}\right]=\left[\begin{array}{ccc|cc|c|c|c}-2 & 10 & 0 & 1 & 0 & 0.5 & 2.5 & 0 \\ 1 & -1 & 1 & 1 & 0 & 0 & 0 & 0.5 \\ 0 & -12 & 0 & 0 & 1 & 1 & -0.1 & 1 \\ \hline-1.5 & 12 & 0 & 0.1 & 0 & 0.8 & 2 & 0 \\ 1 & -1 & 0.9 & 1 & 0 & 0 & 0 & 0.5 \\ 0 & -12 & 0 & 0 & 1 & 1 & -0.1 & 1 \\ \hline-2 & 9 & 0 & 1 & 0 & 0.4 & 1.5 & 0 \\ 0.9 & -1 & 1 & 1 & 0 & 0 & 0 & 0.5 \\ 0 & -11 & 0 & 0 & 1 & 1 & -0.1 & 1 \\ \hline-1.5 & 10 & 0 & 0.1 & 0 & 0.8 & 2.5 & 0 \\ 0.6 & -1 & 0.6 & 1 & 0 & 0 & 0 & 0.5 \\ 0 & -10 & 0 & 0 & 1 & 1 & -0.5 & 1\end{array}\right]$.

We assume that the nonlinearity $\phi_{i}(\cdot)$ for all $i=1,2,3,4$ is sector bounded with $S_{1}=0$ and $S_{2}=2$. The network is described by Laplacian matrix

$$
L=\left[\begin{array}{cccc}
1 & -1 & 0 & 0 \\
-2 & 2 & 0 & 0 \\
-1 & 0 & 1 & 0 \\
0 & -1 & -1 & 2
\end{array}\right]
$$

We firstly specify the synchronization pattern (3) by $A_{0}=\left[\begin{array}{cc}0 & 1 \\ -1 & 0\end{array}\right]$ and $C_{0}=\left[\begin{array}{ll}1 & 0\end{array}\right]$. Referring to the classic monograph [35] on solving linear regulator equations, we can easily find a set of solution pairs

$$
\left\{\begin{array}{c}
{\left[\begin{array}{c|c|cc}
\Pi_{1} & \Pi_{2} & \Pi_{3} & \Pi_{4}
\end{array}\right]} \\
=\left[\begin{array}{cc|cc}
2.7059 & 0.2353 & 1.3586 & 0.0752 \\
0.7059 & 0.2353 & 0.1738 & 0.1203 \\
-0.3529 & -0.1176 & -0.0869 & -0.0602
\end{array} \mid\right.
\end{array}\right.
$$




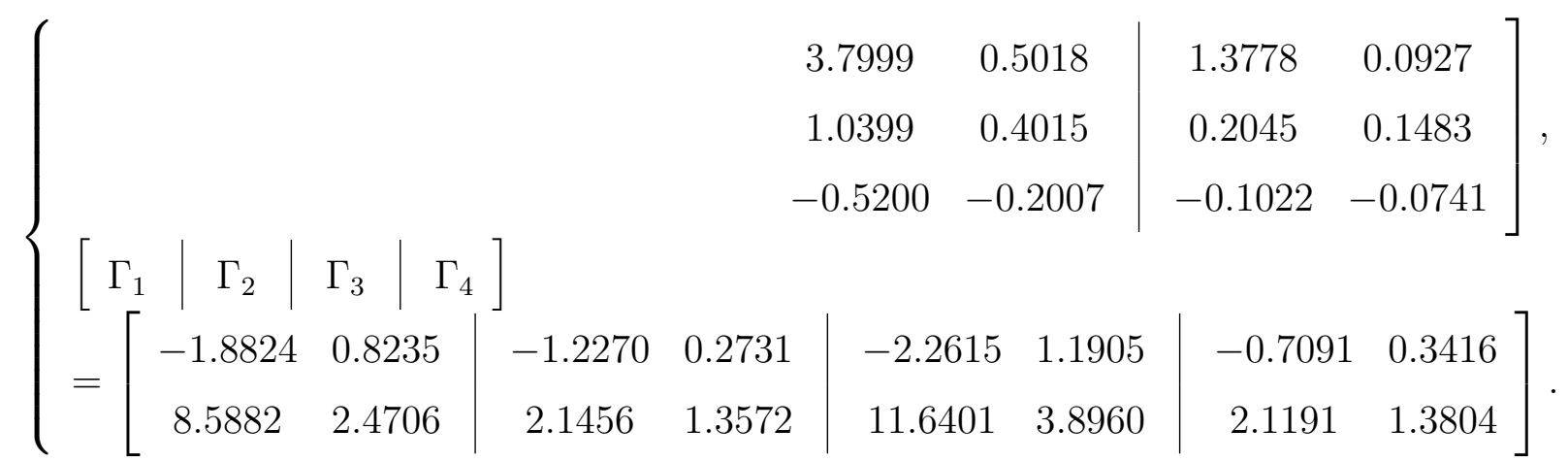

After these preparations, we resort to Theorem 3.2 and Corollary 3.3, along with the iterative algorithm, to compute the state-feedback controller and observer-based controller as follows,

$$
\begin{aligned}
& \left\{\begin{array}{l}
{\left[\begin{array}{l|l|l|lll}
K_{11} & K_{12} & K_{13} & K_{14}
\end{array}\right]} \\
=\left[\begin{array}{ccc|ccc}
-6.6589 & -10.8108 & -2.2624 & -6.9472 & -17.8212 & -2.0693 \\
2.6389 & 12.8915 & -9.2978 & 2.4400 & 16.0233 & -9.6740
\end{array}\right)
\end{array}\right. \\
& \left.\begin{array}{ccc|ccc}
-7.4437 & -9.6917 & -1.4261 & -7.6034 & -17.7449 & -2.5003 \\
3.0985 & 11.3786 & -9.7294 & 3.1593 & 14.3198 & -8.7416
\end{array}\right], \\
& {\left[\begin{array}{l|l|l|l}
K_{21} & K_{22} & K_{23} & K_{24}
\end{array}\right]} \\
& \left\{=\left[\begin{array}{cc|cc}
18.3006 & 27.6363 & 8.3130 & 13.9446 \\
-8.6563 & -13.2113 & -3.4580 & -6.1314
\end{array} \mid\right.\right.
\end{aligned}
$$

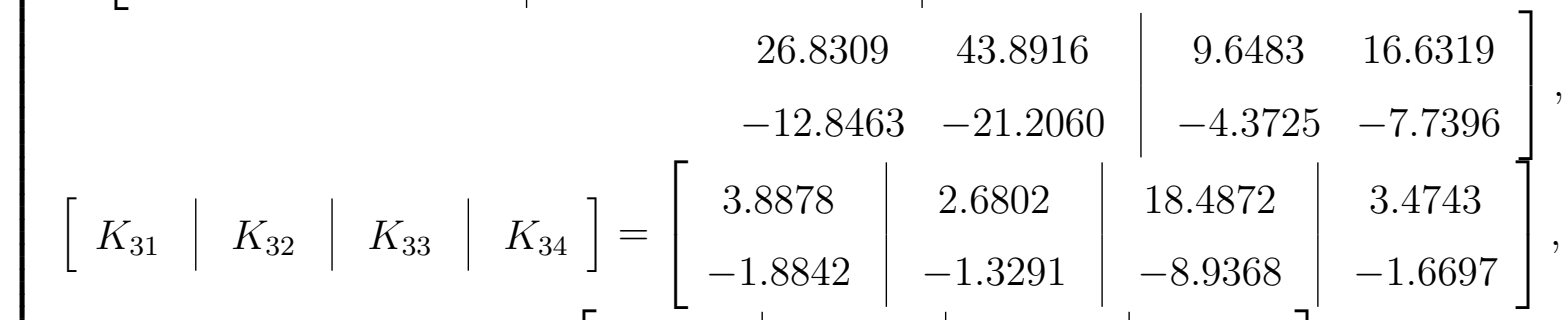

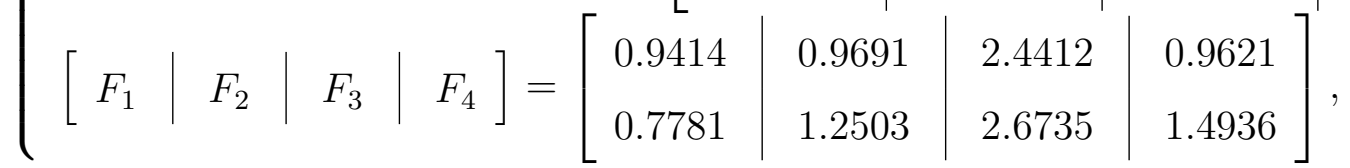

for the state-feedback controller, and

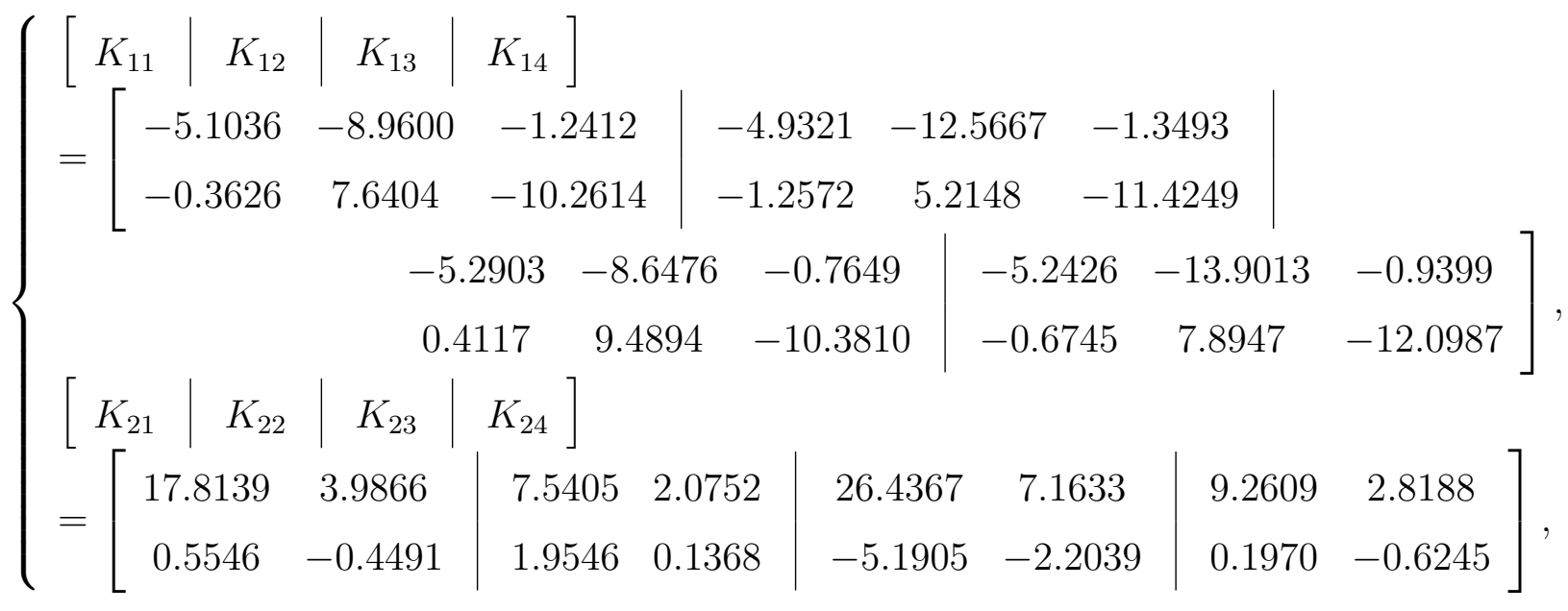




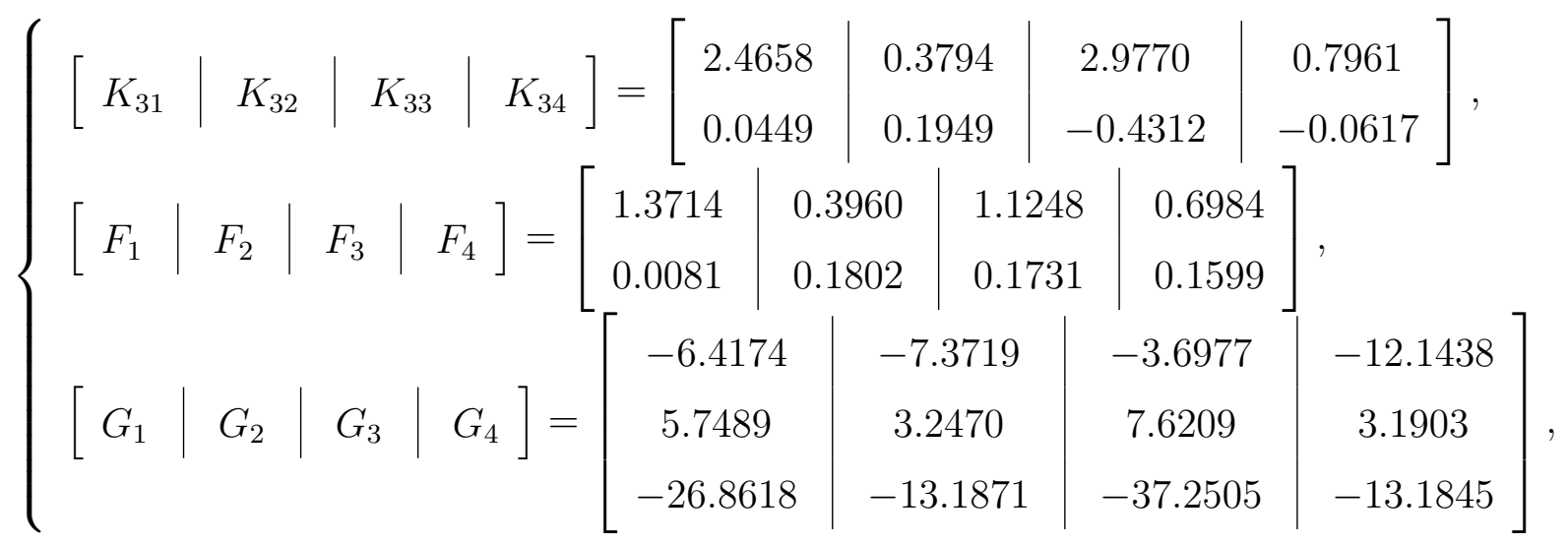

for the observer-based controller.

As we focus on the output synchronization problem for heterogeneous networks, the agent outputs should own a uniform dimension to ensure the output synchronization. However, the dimensions of system states and inputs are not constrained. In particular, we will provide another numerical example with different dimensions of agent states and control inputs to illustrate the theoretical results.

Example 4.2. Consider another group of four agents in the form of (1), where the system parameters are given as below,

$\left[\begin{array}{c|c|c|c|c}A_{1} & B_{1} & C_{1} & E_{1} & H_{1} \\ \hline A_{2} & B_{2} & C_{2} & E_{2} & H_{2} \\ \hline A_{3} & B_{3} & C_{3} & E_{3} & H_{3} \\ \hline A_{4} & B_{4} & C_{4} & E_{4} & H_{4}\end{array}\right]$

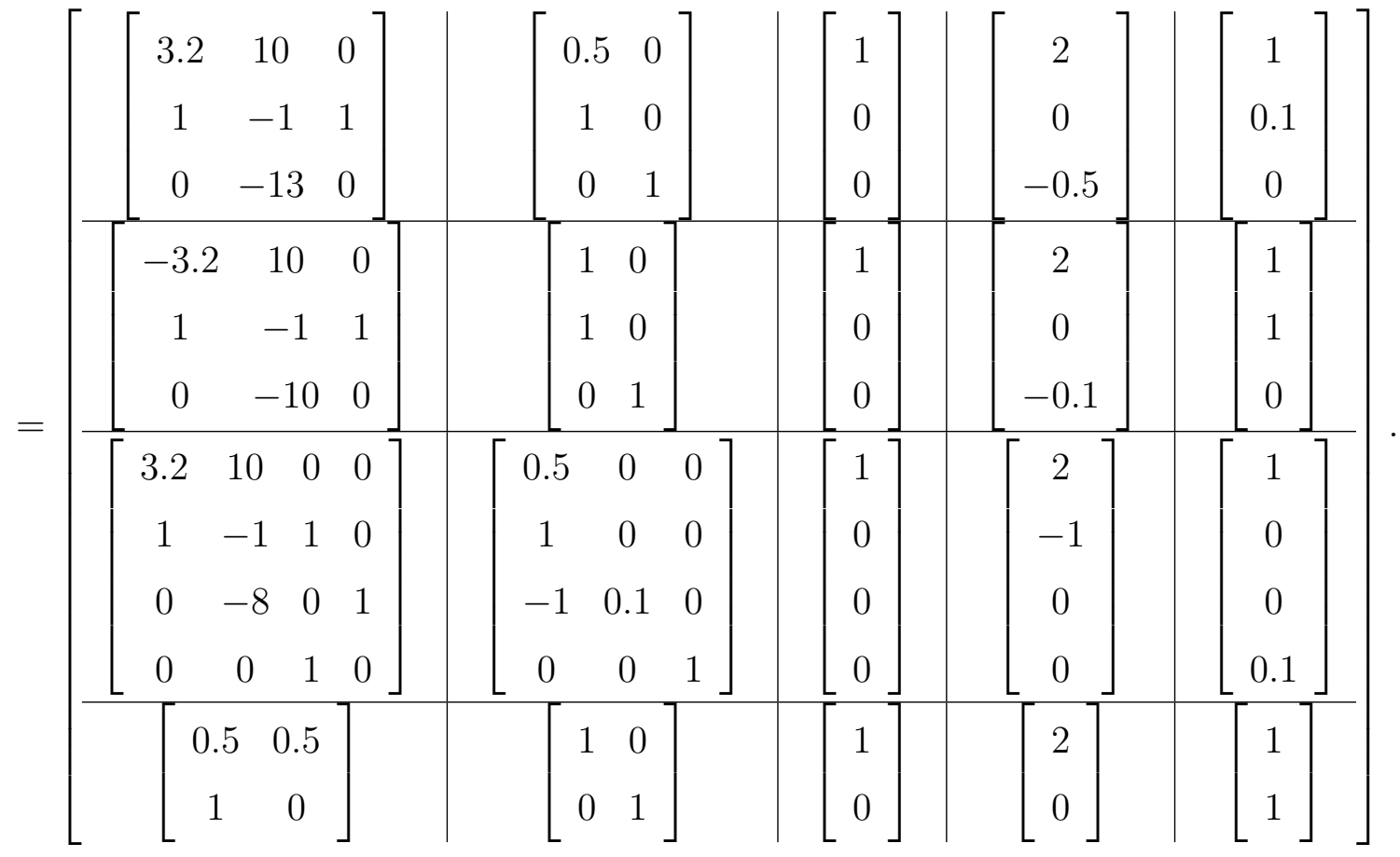


The nonlinearity $\phi_{i}(\cdot)$ for each agent $i$ is described by $(0.5 i+1) \operatorname{atan}(\cdot), i=1,2,3,4$, where atan refers to the arctangent function. It can be readily checked as shown in Fig. 1 that the function $(0.5 i+1) \operatorname{atan}(\cdot), i=1,2,3,4$ is sector bounded with $S_{1}=0$ and $S_{2}=8$. The Laplacian matrix $L$ of network configuration is selected as in (29), and the parameters, i.e., $A_{0}$ and $C_{0}$, of the synchronization pattern are also borrowed from Example 4.1. Similarly, we can search for a set of solution pairs

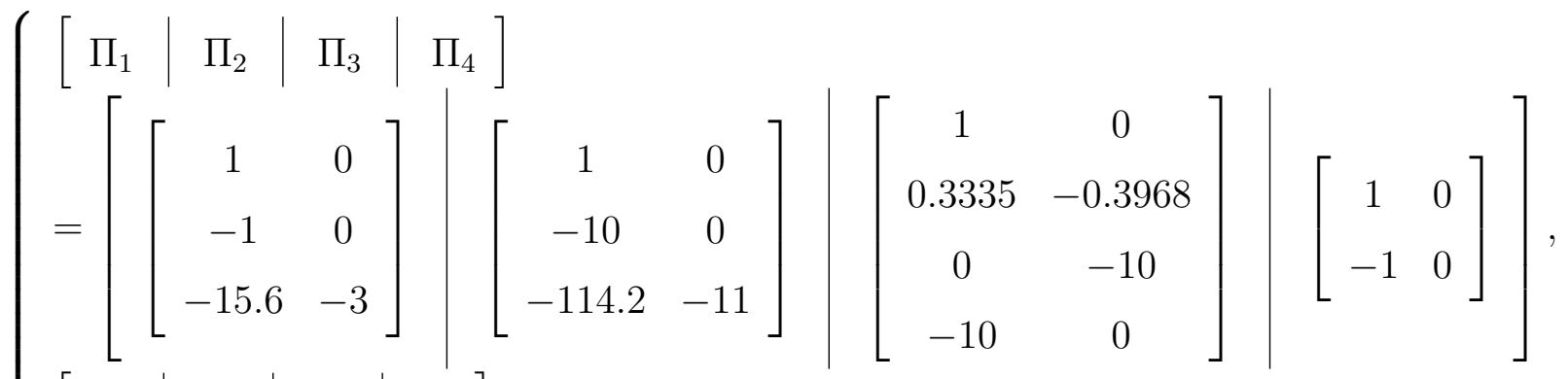

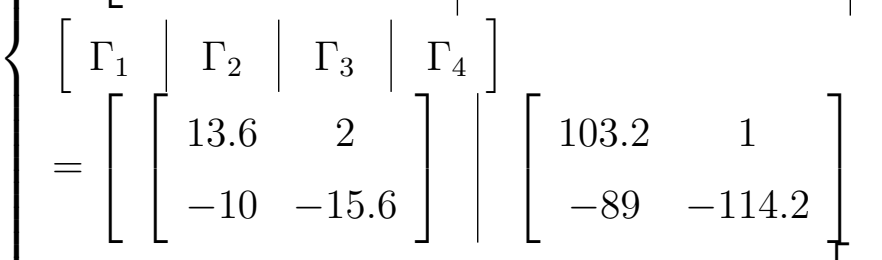

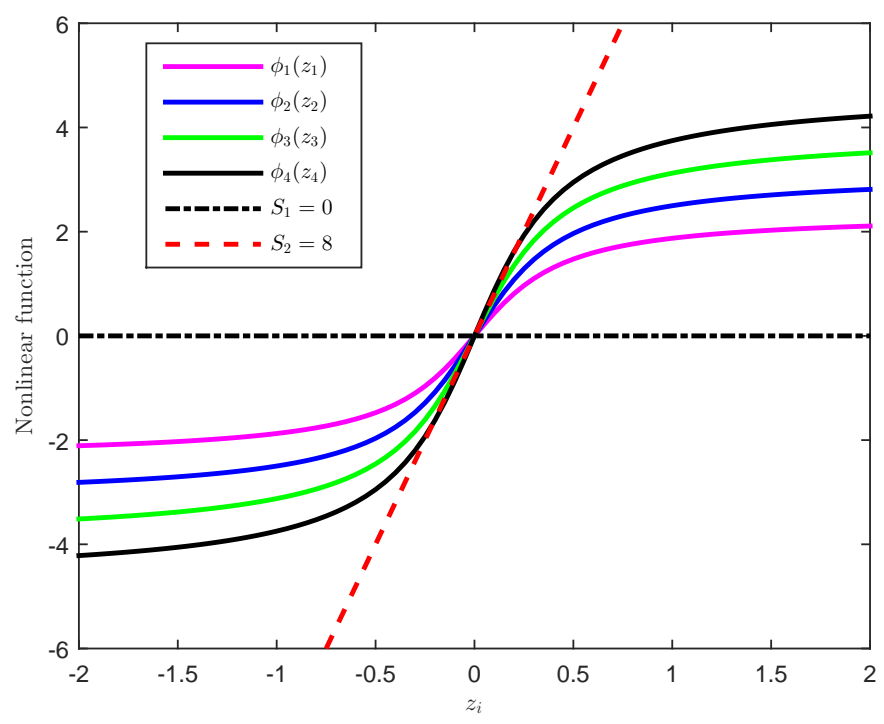

Fig. 1: The nonlinear function $\phi_{i}(\cdot)$ 
Then, solving the iterative algorithm referring to Theorem 3.2 yields the distributed state-feedback control parameters as follows,

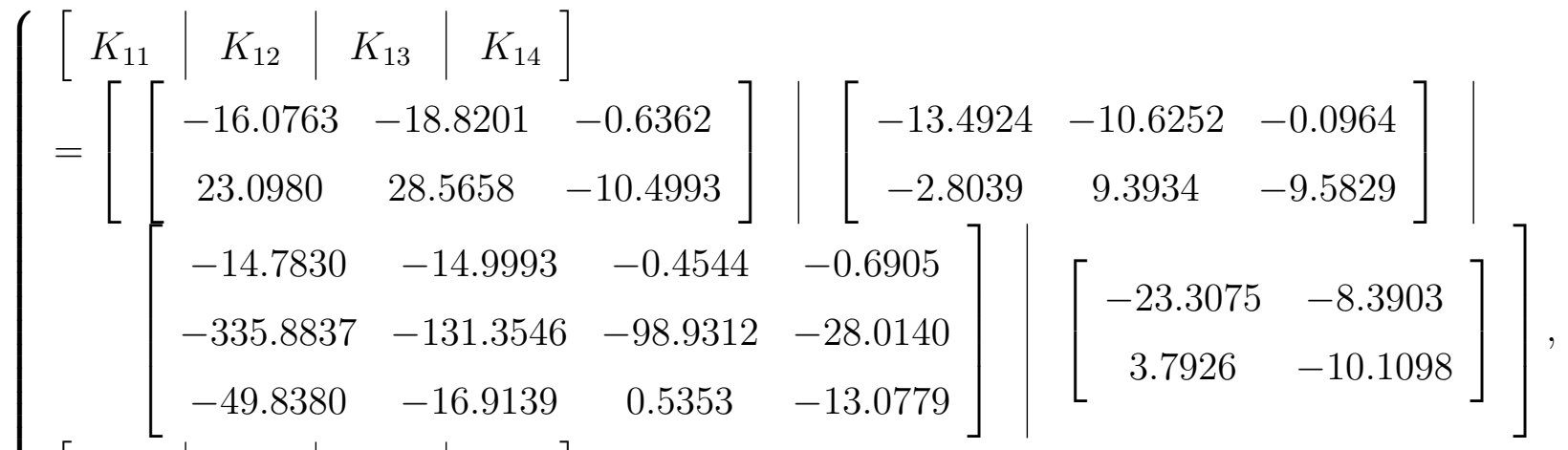

$$
\begin{aligned}
& {\left[\begin{array}{l|l|l|l}
K_{21} & K_{22} & K_{23} & K_{24}
\end{array}\right]} \\
& =\left[\begin{array}{cc}
0.8398 & 1.0225 \\
-121.2237 & -215.4196
\end{array}\right] \mid\left[\begin{array}{cc}
0.5 & 0.6 \\
-867 & -1306.2
\end{array}\right] \\
& \left.\left[\begin{array}{cc}
13.2 & 12.1 \\
1297.3 & -650.3 \\
-73.9 & -76.7
\end{array}\right] \quad\left[\begin{array}{cc}
13.9172 & 15.9172 \\
-13.9024 & -15.9024
\end{array}\right]\right] \\
& {\left[\begin{array}{l|l|l|l}
K_{31} & K_{32} & K_{33} & K_{34}
\end{array}\right]} \\
& \left.=\left[\begin{array}{c}
0.0733 \\
-17.2047
\end{array}\right]\left|\left[\begin{array}{c}
-0.0391 \\
-85.7026
\end{array}\right]\right|\left[\begin{array}{c}
1.8313 \\
-80.4583 \\
-11.4220
\end{array}\right] \mid\left[\begin{array}{c}
2.5005 \\
-2.5024
\end{array}\right]\right] \text {, } \\
& {\left[\begin{array}{l|l|l|l}
F_{1} & F_{2} & F_{3} & F_{4}
\end{array}\right]=\left[\begin{array}{l|l|l|l}
0.4953 & 0.2681 & 0.2350 & 1.5489 \\
0.5042 & 0.5046 & 1.3874 & 1.7131
\end{array}\right] \text {. }}
\end{aligned}
$$

To further verify the effectiveness of the design procedure, simulations are conducted with a series of initial conditions. For example, for initial conditions

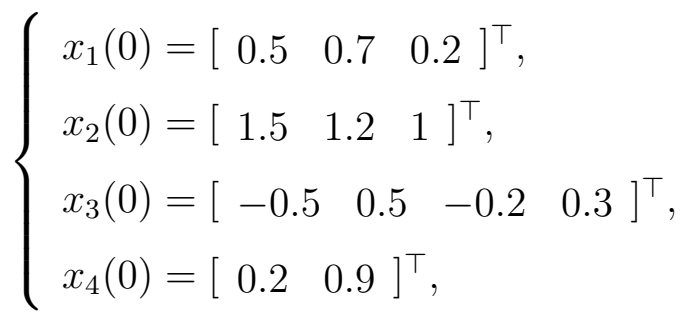

and

$$
\left\{\begin{array}{l}
\hat{x}_{1}(0)=\left[\begin{array}{ll}
1 & 0.5
\end{array}\right]^{\top} \\
\hat{x}_{2}(0)=\left[\begin{array}{ll}
2 & 0.5
\end{array}\right]^{\top} \\
\hat{x}_{3}(0)=\left[\begin{array}{ll}
2 & 2
\end{array}\right]^{\top} \\
\hat{x}_{4}(0)=\left[\begin{array}{ll}
1 & 1
\end{array}\right]^{\top}
\end{array}\right.
$$




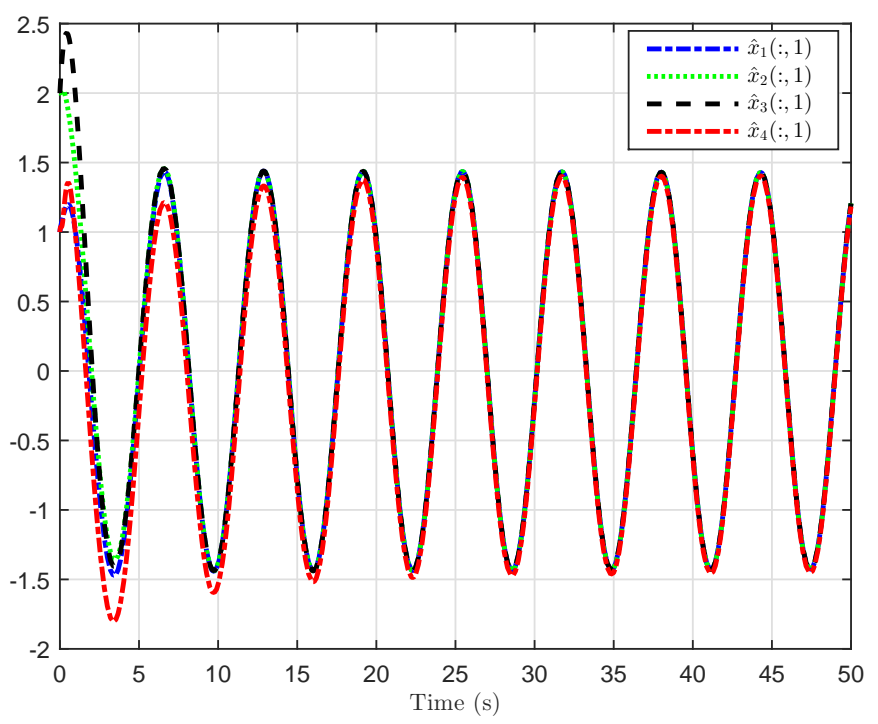

Fig. 2: The first components of dynamic compensators' state variables $\hat{x}_{i}, i=1,2,3,4$

the simulation results are shown in Figs. 2 and 3, showing that the agent outputs $y_{i}, i=1,2,3,4$, synchronize to the common trajectory in a desired pattern.

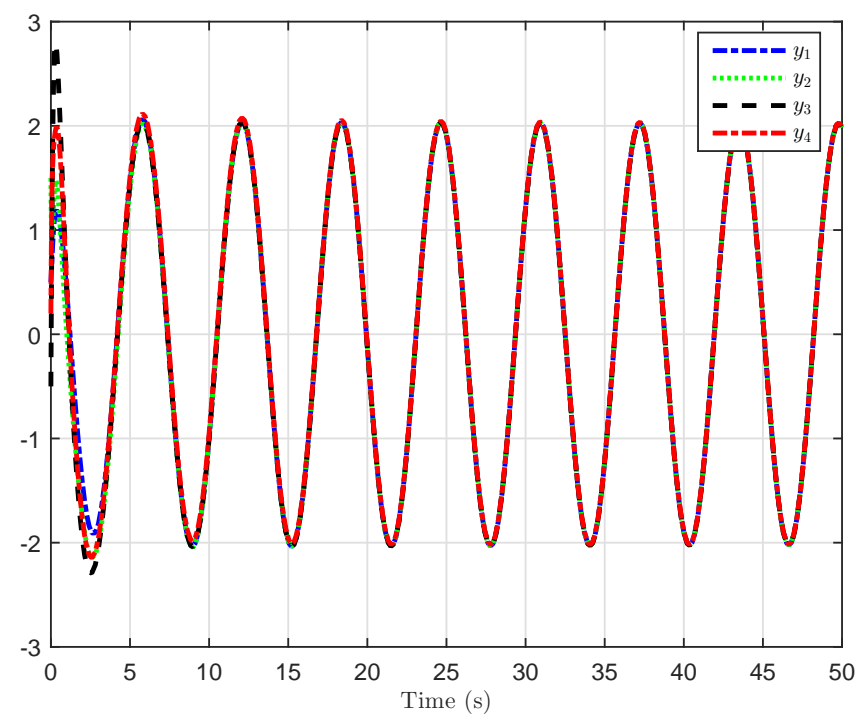

Fig. 3: The agent outputs synchronization pattern 


\section{CONCLUSIONS}

In this paper, we have studied the output synchronization problem for heterogeneous multiagent systems with nonlinear dynamics. We first presented a state-feedback controlller, where synchrnozation is inferred from a combination of local output regulation, based on the internal model principle, and diffusive output coupling between the agents. A distinctive feature of the control strategy is that the synchronization is achieved in a leader-free framework and the communication of the internal model information is avoided, resulting in a distributed control structure. Therefore, it not only reduces the communication burden but it also eases the implementation of the controller. Both state feedback and observer-based controllers were considered, and an iterative procedure for computing the controller matrices was presented. It has been shown that the output synchronization problem can be addressed by the proposed controllers, where the control gains can be synthesized using an iterative algorithm.

It is noted that the generalization of the proposed method to the output synchronization of heterogeneous nonlinear networks with communication delays deserves further investigation [40]-[42]. In addition, it is also interesting to explore the containment control problem of heterogeneous nonlinear networks with multiple leaders [44], [45].

\section{APPENDIX}

Proof of Corollary 3.2: As for the output synchronization error in (8), we shall focus on the dynamics of $e_{x}(t):=x(t)-\Pi \hat{x}(t)$ and $e_{\hat{x}}(t):=\left(U^{\top} \otimes \mathbf{I}\right) \hat{x}(t)$. Similarly to the calculations on state-feedback case, we obtain,

$$
\begin{aligned}
\dot{e}_{x}(t)= & \hat{\mathcal{A}}_{x} x(t)+\hat{\mathcal{A}}_{\xi} \xi(t)+\hat{\mathcal{A}}_{\hat{x}} \hat{x}(t)-\Pi\left[\left(\mathbf{I}_{N} \otimes A_{0}\right) \hat{x}(t)+\overline{\mathcal{D}} x(t)\right]+\mathcal{E} \phi(z) \\
= & \hat{\mathcal{A}}_{x} x(t)+\hat{\mathcal{A}}_{\xi} \xi(t)+\hat{\mathcal{A}}_{\hat{x}} \hat{x}(t)-\Pi \overline{\mathcal{D}} x(t)-\left[\left(\mathcal{A}+\mathcal{B} \mathcal{K}_{1}\right) \Pi+\mathcal{B} \mathcal{K}_{2}\right] \hat{x}(t)+\mathcal{E} \phi(z) \\
= & \left(\mathcal{A}+\mathcal{B} \mathcal{K}_{1}\right) e_{x}(t)-\left(\mathcal{B \mathcal { K }}_{3}-\Pi \mathcal{F}\right)(L \otimes \mathbf{I}) \mathcal{C} x(t)-\mathcal{B} \mathcal{K}_{1} e_{\xi}(t)+\mathcal{E} \phi(z) \\
= & \left(\mathcal{A}+\mathcal{B} \mathcal{K}_{1}\right) e_{x}(t)-\left(\mathcal{B \mathcal { K }}_{3}-\Pi \mathcal{F}\right)\left(L\left(\mathbf{I}-\frac{1}{N} \mathbf{1}_{N} \mathbf{1}_{N}^{\top}\right) \otimes \mathbf{I}\right) \mathcal{C} x(t)-\mathcal{B} \mathcal{K}_{1} e_{\xi}(t)+\mathcal{E} \phi(z) \\
= & {\left[\mathcal{A}+\mathcal{B} \mathcal{K}_{1}-\left(\mathcal{B} \mathcal{K}_{3}-\Pi \mathcal{F}\right)(L \otimes \mathbf{I}) \mathcal{C}\right] e_{x}(t) } \\
& \quad-\left(\mathcal{B} \mathcal{K}_{3}-\Pi \mathcal{F}\right)\left(L U \otimes C_{0}\right) e_{\hat{x}}(t)-\mathcal{B K}_{1} e_{\xi}(t)+\mathcal{E} \phi(z),
\end{aligned}
$$

with $e_{\xi}(t):=x(t)-\xi(t)$, and

$$
\begin{aligned}
\dot{e}_{\hat{x}}(t) & =\left(U^{\top} \otimes \mathbf{I}\right)\left[\left(\mathbf{I} \otimes A_{0}\right) \hat{x}(t)+\overline{\mathcal{D}} x(t)\right] \\
& =\left[\mathbf{I} \otimes A_{0}-\left(U^{\top} \otimes \mathbf{I}\right) \mathcal{F}\left(L U \otimes C_{0}\right)\right] e_{\hat{x}}(t)-\left(U^{\top} \otimes \mathbf{I}\right) \mathcal{F}(L \otimes \mathbf{I}) \mathcal{C} e_{x}(t),
\end{aligned}
$$

where we utilize the conditions in the regulator equation (5). 
In the observer-based dynamic feedback framework, the evolution of the synchronization error $e_{y}(t)$ of agents actually is determined by,

$$
\left\{\begin{aligned}
& \dot{e}_{x}(t)=\left[\mathcal{A}+\mathcal{B} \mathcal{K}_{1}-\left(\mathcal{B} \mathcal{K}_{3}-\Pi \mathcal{F}\right)(L \otimes \mathbf{I}) \mathcal{C}\right] e_{x}(t) \\
&-\left(\mathcal{B K} \mathcal{K}_{3}-\Pi \mathcal{F}\right)\left(L U \otimes C_{0}\right) e_{\hat{x}}(t)-\mathcal{B} \mathcal{K}_{1} e_{\xi}(t)+\mathcal{E} \phi\left(\mathcal{H} e_{x}\right), \\
& \dot{e}_{\hat{x}}(t)=\left[\mathbf{I} \otimes A_{0}-\left(U^{\top} \otimes \mathbf{I}\right) \mathcal{F}\left(L U \otimes C_{0}\right)\right] e_{\hat{x}}(t)-\left(U^{\top} \otimes \mathbf{I}\right) \mathcal{F}(L \otimes \mathbf{I}) \mathcal{C} e_{x}(t), \\
& \dot{e}_{\xi}(t)=(\mathcal{A}+\mathcal{G C}) e_{\xi}(t)+\mathcal{E} \phi\left(\mathcal{H} e_{x}\right) .
\end{aligned}\right.
$$

Likely, we choose the Lyapunov function $V\left(e_{x}, e_{\hat{x}}, e_{\xi}\right)=\left[\begin{array}{lll}e_{x}^{\top} & e_{\hat{x}}^{\top} & e_{\xi}^{\top}\end{array}\right]^{\top} \bar{P}\left[\begin{array}{lll}e_{x} & e_{\hat{x}} & e_{\xi}\end{array}\right]$ with $\bar{P}>0$. It can be readily checked that $V\left(e_{x}, e_{\hat{x}}, e_{\xi}\right)$ is positive definite and radially unbounded. Then evaluating the time derivative of $V\left(e_{x}, e_{\hat{x}}, e_{\xi}\right)$ along the trajectories of the error system (32), we obtain,

$$
\dot{V}\left(e_{x}, e_{\hat{x}}, e_{\xi}\right)=2\left[\begin{array}{lll}
e_{x}^{\top} & e_{\hat{x}}^{\top} & e_{\xi}^{\top}
\end{array}\right]^{\top} \bar{P}\left[\overline{\mathscr{A}}\left[\begin{array}{lll}
e_{x} & e_{\hat{x}} & e_{\xi}
\end{array}\right]+\overline{\mathscr{E}} \phi\left(\mathcal{H} e_{x}\right)\right]
$$

where $\overline{\mathscr{A}}$ and $\overline{\mathscr{E}}$ are defined in (27). This, together with sector bounded condition (13), leads us to the conclusion. The proof is thus completed. $\diamond$

\section{REFERENCES}

[1] T. Vicsek, A. Czirók, E. Ben-Jacob, I. Cohen, and O. Shochet, "Novel type of phase transition in a system of self-driven particles," Physical Review Letters, vol. 75, pp. 1226-1229, Aug. 1995.

[2] J. Toner and Y. Tu, "Flocks, herds, and schools: a quantitative theory of flocking," Physical Review E, vol. 58, pp. 4828-4858, Oct. 1998.

[3] C. L. Philip Chen, Y. Liu, and G. Wen, "Fuzzy neural network-based adaptive control for a class of uncertain nonlinear stochastic systems," IEEE Transactions on Cybernetics, vol. 44, no. 5, pp. 583-593, May 2014.

[4] W. Michiels and H. Nijmeijer, "Synchronization of delay-coupled nonlinear oscillators: An approach based on the stability analysis of synchronized equilibria," Chaos: An Interdisciplinary Journal of Nonlinear Science, vol. 19, no. 3, pp. 033110, Sep. 2009.

[5] H. Liang, Y. Zhang, T. Huang, and H. Ma, "Prescribed performance cooperative control for multiagent systems with input quantization," IEEE Transactions on Cybernetics, doi: 10.1109/TCYB.2019.2893645, Jan. 2019.

[6] H. Yan, C. Hu, H. Zhang, H. R. Karimi. X. Jiang, and M. Liu, " $H_{\infty}$ output tracking control for networked systems with adaptively adjusted event-triggered scheme," IEEE Transactions on Systems, Man, and Cybernetics: Systems, doi: 10.1109/TSMC.2017.2788187, Jan. 2018.

[7] H. Zhang, Z. Wang, H. Yan, F. Yang, X. Zhou, "Adaptive event-triggered transmission scheme and $H_{\infty}$ filtering co-design over a filtering network with switching topology," IEEE Transactions on Cybernetics, doi: 10.1109/TCYB.2018.2862828, Sep. 2018.

[8] H. Li, Y. Gao, P. Shi, and H. K. Lam, "Observer-based fault detection for nonlinear systems with sensor fault and limited communication capacity," IEEE Transactions on Automatic Control, vol. 61, no. 9, pp. 2745-2751, Sep. 2016.

[9] H. Li, Z. Chen, L. Wu, and H. K. Lam, "Event-triggered control for nonlinear systems under unreliable communication links," IEEE Transactions on Fuzzy Systems, vol. 25, no. 4, pp. 813-824, Aug. 2017. 
[10] Y. Su, and J. Huang, "Cooperative global robust output regulation for nonlinear uncertain multi-Agent systems in lower triangular form,” IEEE Transactions on Automatic Control, vol. 60, no. 9, pp. 2378-2379, Sep. 2015.

[11] W. Liu, and J. Huang, "Cooperative global robust output regulation for a class of nonlinear multi-agent systems with switching network," IEEE Transactions on Automatic Control, vol. 60, no. 7, pp. 1963-1968, Jul. 2015.

[12] L. Scardovi and R. Sepulchre, "Synchronization in networks of identical linear systems," Automatica, vol. 45, no. 11, pp. 2557-2562, Nov. 2009.

[13] K. Hengster-Movric, F. L. Lewis, and M. Sebek, "Distributed static output-feedback control for state synchronization in networks of identical LTI systems," Automatica, vol. 53, no. 3, pp. 282-290, Mar. 2015.

[14] H. F. Grip, A. Saberi, and A. A. Stoorvogel, "Synchronization in networks of minimum-phase, non-introspective agents without exchange of controller states: homogeneous, heterogeneous, and nonlinear," Automatica, vol. 54, pp. 246-255, 2015.

[15] A. Wahrburg and J. Adamy, "Observer-based synchronization of heterogeneous multi-agent systems by homogenization," in Australian Control Conference, Nov. 2011.

[16] L. Zhu, Z. Chen, and R. Middleton, "Robust asymptotic model matching and its application to output synchronization of heterogeneous multi-agent systems," Systems \& Control Letters, vol. 94, pp.37-45, Aug. 2016.

[17] Y. Su, Y. Hong, and J. Huang, "A general result on the robust cooperative output regulation for linear uncertain multi-agent systems. IEEE Transactions on Automatic Control, vol. 58, no. 5, pp. 1275-1279, May 2013.

[18] Z. Meng, T. Yang, D. V. Dimarogonas, and K. H. Johansson, "Coordinated output regulation of heterogeneous linear systems under switching topologies," Automatica, vol. 53, pp. 362-368, 2015.

[19] B. A. Francis and W. M. Wonham, “The internal model principle of control theory,” Automatica, vol. 12, no. 5, pp. 457-465, May 1976.

[20] P. Wieland, R. Sepulchre, F. Allgöwer, "An internal model principle is necessary and sufficient for linear output synchronization," Automatica, vol. 47, no. 5, pp. 1068-1074, May 2011.

[21] W. He, C. Xu, Q.-L. Han, F. Qian, and Z. Lang, "Finite-time $\mathscr{L}_{2}$ leader-follower consensus of networked Euler-Lagrange systems with external disturbances," IEEE Transactions on Systems, Man, and Cybernetics: Systems, vol. 48, no. 11, pp. 1920-1928, Nov. 2018.

[22] W. Yu, G. Chen, and M. Cao, "Consensus in directed networks of agents with nonlinear dynamics," IEEE Transactions on Automatic Control, vol. 56, no. 6, pp. 1436-1441, Jun. 2011.

[23] H. Kim, H. Shim, and J. Seo, "Output consensus of heterogeneous uncertain linear multi-agent systems," IEEE Transactions on Automatic Control, vol. 56, no. 1, pp. 200-206, Jan. 2011.

[24] Z. Qu, J. Chunyu, and J. Wang, "Nonlinear cooperative control for consensus of nonlinear and heterogeneous systems," in 46th IEEE Conference on Decision and Control, pp. 2301-2308, Dec. 2007.

[25] Z. T. Ding, "Consensus output regulation of a class of heterogeneous nonlinear systems," IEEE Transactions on Automatic Control, vol. 58, no. 10, pp. 2648-2653, Oct. 2013.

[26] Y. Dong and J. Huang, "Leader-following connectivity preservation rendezvous of multiple double integrator systems based on position measurement only," IEEE Transactions on Automatic Control, vol. 59, no. 9, pp. 2598-2603, Sep. 2014.

[27] L. Zhu, Z. Chen, and R. H. Middleton, "A general framework for robust output synchronization of heterogeneous nonlinear networked systems," IEEE Transactions on Automatic Control, vol. 61, no. 8, pp. 2092-2107, Aug. 2016.

[28] Q. Liu, M. Ye, J. Qin, and C. Yu, "Event-triggered algorithms for leader-follower consensus of networked Euler-Lagrange agents," IEEE Transactions on Systems, Man, and Cybernetics: Systems, doi: 10.1109/TSMC.2017.2772820, Dec. 2017.

[29] Q. Wang and C. Sun, "Adaptive consensus of multiagent systems with unknown high-frequency gain signs under directed graphs," IEEE Transactions on Systems, Man, and Cybernetics: Systems, doi: 10.1109/TSMC.2018.2810089, Mar. 2018. 
[30] H. Cai, F. L. Lewis, G. Hu, and J. Huang, "The adaptive distributed observer approach to the cooperative output regulation of linear multi-agent systems," Automatica, vol. 75, pp. 299-305, 2017.

[31] H. Cai and J. Huang, "The leader-following consensus for multiple uncertain Euler-Lagrange systems with an adaptive distributed observer," IEEE Transactions on Automatic Control, vol. 61, no. 10, pp. 3152-3157, Oct. 2016.

[32] P. Zhou, S. Cai, J. Shen, and Z. Liu, "Adaptive exponential cluster synchronization in colored community networks via aperiodically intermittent pinning control," Nonlinear Dynamics, vol. 92, no. 3, pp. 905-921, May 2016.

[33] F. L. Lewis, B. Cui, T. Ma, Y. Song, and C. Zhao, "Heterogeneous multi-agent systems: reduced-order synchronization and geometry," IEEE Transactions on Automatic Control, vol. 61, no. 5, pp. 1391-1396, May 2016.

[34] C. Godsil and G. Royle, Algebraic Graph Theory. New York: Springer-Verlag, 2001.

[35] J. Huang, Nonlinear Output Regulation: Theory and Applications. Philadelphia: Society for Industrial and Applied Mathematics, 2004.

[36] A. Pavlov, N. van de Wouw, and H. Nijmeijer, "Global nonlinear output regulation: convergence-based controller design," Automatica, vol. 43, no. 3, pp. 456-463, Mar. 2007.

[37] Y. Wu and R. Lu, "Output synchronization and $\mathscr{L}_{2}$-gain analysis for network systems," IEEE Transactions on Systems, Man, and Cybernetics: Systems, vol. 48, no. 12, pp. 2105-2114, Dec. 2018.

[38] L. El Ghaoui, F. Oustry, and M. AitRami, "A cone complementarity linearization algorithm for static output-feedback and related problem," IEEE Transactions on Automatic Control, vol. 42, no. 8, pp. 1171-1176, Aug. 1997.

[39] S. P. Boyd, L. El Ghaoui, E. Feron, and V. Balakrishnan, Linear Matrix Inequalities in System and Control Theory, Philadelphia. PA: SIAM, 1994.

[40] R. Yang, H. Zhang, G. Feng, H. Yan, and Z. Wang, "Robust cooperative output regulation of multi-agent systems via adaptive event-triggered control," Automatica, vol. 102, pp. 129-136, Apr. 2019.

[41] H. Yan, Y. Tian, H. Li, H. Zhang, and Z. Li, "Input-output finite-time mean square stabilization of nonlinear semi-Markovian jump systems," Automatica, vol. 104, pp. 82-89, Jun. 2019.

[42] L. Xiong, J. Cheng, J. Cao, and Z. Liu, "Novel inequality with application to improve the stability criterion for dynamical systems with two additive time-varying delays," Applied Mathematics and Computation, vol. 321, pp. 672-688, Mar. 2018.

[43] X. Xie, Q. Zhou, D. Yue, and H. Li, "Relaxed control design of discrete-time Takagi-Sugeno fuzzy systems: An eventtriggered real-time scheduling approach,” IEEE Transactions on Systems, Man, and Cybernetics: Systems, vol. 48, no. 12, pp. 2251-2262, Dec. 2018.

[44] H. Liang, Y. Zhou, H. Ma, and Q. Zhou, "Adaptive distributed observer approach for cooperative containment control of nonidentical networks," IEEE Transactions on Systems, Man, and Cybernetics: Systems, vol. 49, no. 2, pp. 299-307, Feb. 2019.

[45] H. Yan, X. Zhou, H. Zhang, F. Yang, and Z. Wu, "A novel sliding mode estimation for microgrid with time delays based on power line communication," IEEE Transactions on Smart Grid, vol. 10, no. 2, pp. 1509-1520, Mar. 2019. 